BMJ Nutrition,

Prevention \& Health

\section{How fragile are Mediterranean diet interventions? A research-on-research study of randomised controlled trials}

To cite: Grammatikopoulou MG, Nigdelis MP, Theodoridis X, et al. How fragile are Mediterranean diet interventions? A research-onresearch study of randomised controlled trials. BMJ Nutrition, Prevention \& Health 2021;4:e000188. doi:10.1136/ bmjnph-2020-000188

For numbered affiliations see end of article.

\section{Correspondence to}

Dr Maria G Grammatikopoulou,

Nutritional Sciences \&

Dieteticss, International Hellenic University Faculty of Health Sciences, Alexander Campus, Thessaloniki, Greece; mariagram@auth.gr and

Professor Dimitrios G Goulis, Unit of Reproductive Endocrinology, 1st Department of Obstetrics and Gynecology, Aristotle University of Thessaloniki Faculty of Health Sciences, Thessaloniki, Greece; dgg@auth.gr

MPN and XT contributed equally.

Received 30 September 2020 Revised 4 February 2021 Accepted 13 February 2021

Published Online First 9 March 2021

Check for updates

(c) Author(s) (or their employer(s)) 2021. Re-use permitted under CC BY-NC. No commercial re-use. See rights and permissions. Published by BMJ.

\section{ABSTRACT}

Introduction The Mediterranean diet (MD) is a traditional regional dietary pattern and a healthy diet recommended for the primary and secondary prevention of various diseases and health conditions. Results from the higher level of primary evidence, namely randomised controlled trials (RCTs), are often used to produce dietary recommendations; however, the robustness of RCTs with MD interventions is unknown.

Methods A systematic search was conducted and all MD RCTs with dichotomous primary outcomes were extracted from PubMed. The fragility (FI) and the reverse fragility index (RFI) were calculated for the trials with significant and non-significant comparisons, respectively.

Results Out of 27 RCTs of parallel design, the majority failed to present a significant primary outcome, exhibiting an Fl equal to 0 . The median $\mathrm{Fl}$ of the significant comparisons was 5 , ranging between 1 and 39 . More than half of the comparisons had an $\mathrm{FI}<5$, indicating that the addition of 1-4 events to the treatment arm eliminated the statistical significance. For the comparisons with an $\mathrm{Fl}=0$, the RFI ranged between 1 and 29 (Median RFI: 7). When the included RCTs were stratified according to masking, the use of a composite primary endpoint, sample size, outcome category, or dietary adherence assessment method, no differences were exhibited in the FI and RFI between groups, except for the RFI among different compliance assessment methods.

Conclusions In essence, the present study shows that even in the top tiers of evidence hierarchy, research on the MD may lack robustness, setting concerns for the formulation of nutrition recommendations.

\section{BACKGROUND}

Since Keys first presented a diet-mortality hypothesis explaining the Seven Countries study results in $1986,{ }^{1}$ the Mediterranean diet (MD) has become a dietary pattern of particular interest. Research on the MD has spiralled, ${ }^{2}{ }^{3}$ reputed for its health effects, spanning from ameliorated cardiovascular disease (CVD) factors, ${ }^{4-7}$ to improved pregnancy outcomes, ${ }^{8}$ ticking all the boxes in the quest for health attainment. For some, the MD is much more than a traditional
What this paper adds

- Recommendations for the adoption of the Mediterranean diet (MD) for improved health outcomes are based mainly on randomised controlled trials (RCTs) and their synthesis.

- The robustness of RCTs with MD interventions appears to be low to moderate. Similarly, fragility (Fls) and reverse fragility indexes (RFIs) have also been reported among RCTs in other therapeutic domains, including clinical nutrition, anesthesiology, perioperative medicine, etc.

- The Fl and RFI can be used to improve and promote the science of nutrition.

regional dietary pattern, being regarded as the 'unicorn' of diet paradigms, with many clinical practice guidelines endorsing the adherence of the MD. ${ }^{9}{ }^{10}$

Apart from many 'followers' however, several scientists are also questioning the MD. Some are high-lightening the observational design of the Seven Countries study, ${ }^{11}$ while others are stressing the limitations of nutritional epidemiology in general, ${ }^{12}$ often incorporating selective reporting, ${ }^{13}$ inflated results, ${ }^{14}$ over-interpretation and skewed perspectives, ${ }^{15}$ with large flexibility in the performed analyses which can be based on questionnaires of low reproducibility. ${ }^{16}$

Subsequently, research designs were improved to minimise bias, ${ }^{14}$ and the focus shifted to randomised controlled trials (RCTs), situated higher in the pyramid of evidence. ${ }^{17}$ The worm turned again when the biggest and most promising MD trial to date, the Prevención con Dieta Mediterránea (PREDIMED), ${ }^{18} 19$ raised concerns over randomisation bias, resulting in its reanalysis. ${ }^{20}$ Nutrition RCTs were once more in the spotlight, and scepticism was apparent, ${ }^{21}$ with researchers questioning the suitability of RCTs for nutrition research and the quality 
of the trials. Most trials tend to report positive findings ${ }^{21}$; however, statistical significance ( $P$-value) does not ensure the robustness of an analysis and a pledge towards the use of more specific measures was made. ${ }^{22-25}$

Today, clinical research continues to emphasise the $P$ threshold of 0.05 when interpreting RCT results. ${ }^{26}$ For this, it is additionally important to evaluate the robustness of RCTs with MD interventions and attain an additional measure of the quality of MD RCTs. Two indexes have been proposed for the evaluation of an RCTs' robustness, ${ }^{27}$ namely the fragility index (FI) and the reverse fragility index (RFI), for trials with significant or nonsignificant findings, respectively. Both indexes can only be calculated on studies with an RCT design and dichotomous primary outcomes.

To assess the robustness of RCTs with MD interventions, the present research-on-research study aimed to identify all RCTs with MD interventions and dichotomous primary outcomes, and calculate their FI or RFI, depending on the significance of the comparisons.

\section{METHODS}

\section{Research question and search strategy}

The present study used a systematic search strategy to answer the question "What is the fragility and reverse fragility index of RCTs assessing MD interventions?" The PICO of the study's hypothesis was P: human population of any age group or health status, I: MD intervention, C: any comparison other than the MD, a sham diet, other diet or no intervention, $\mathrm{O}$ : any dichotomous primary outcome (table 1). To answer this research question, the focus was set on all RCTs examining MD interventions, irrespective of their other characteristics. Similar studies examining the FI/RFI in broad research areas are common in the literature. ${ }^{26}$

The protocol of the study was published at the Center for Open Science https://osf.io/mnx2c/. A systematic search was conducted on PubMed from inception until 31 August 2019, using the keyword (Mediterranean diet) and the PubMed filter for clinical trials.

\section{Inclusion and exclusion criteria}

As the concept of fragility is only applicable to RCTs, only studies with an RCT design were considered eligible. ${ }^{28}$ In parallel, we searched for trials with dichotomous primary

Table 1 PICO strategy of the study's research question

Population Randomised controlled trials performed on humans of any age and health status

$\begin{array}{ll}\text { Intervention } & \text { Mediterranean diet } \\ \text { Comparator(s) } & \begin{array}{l}\text { Any dietary regime other than the } \\ \text { Mediterranean diet, including a sham diet, } \\ \text { nutrient supplementation or no intervention } \\ \text { at all }\end{array}\end{array}$

Outcome(s) Any outcome (perinatal, cardiovascular, metabolic or other) outcomes, as the FI and RFI cannot be calculated in trials with continuous outcomes. Secondary outcomes were not of concern as they are not accounted for when estimating the sample size required for an RCT and should not be used to assess a trial's robustness. ${ }^{29}$ All RCTs with MD interventions were assessed for eligibility, despite other possible heterogeneities, as the research question focused on the FI and RFI of MD interventions in general and not in MD RCTs with more homogenous outcomes/ samples/designs.

The criteria for inclusion in the present analyses involved (1) RCTs performed on humans, (2) of any age group, (3) irrespectively of any medical diagnosis or health condition, (4) applying MD interventions, (5) compared with no intervention, control diet, or to dietary patterns other than the MD, (6) assessing any dichotomous primary outcome and (7) published in any language.

On the other hand, criteria for exclusion involved trials (1) lacking randomisation, (2) performed on animals, (3) with continuous primary outcomes or (4) with dichotomous secondary outcomes, (5) comparing MD interventions to control diets based on the MD, (6) not including an MD intervention, (7) not reporting the number of events and the sample size in each arm, making it impossible to calculate $2 \times 2$ frequency tables, (8) failing to report adequate data to calculate persons-years, (9) trial protocols without results and (10) research performed on animals.

\section{Data extraction}

Two researchers (MGG and XT) independently extracted data from the selected RCTs, aided by an additional pair of reviewers (MPN and KG) when deemed necessary. Extracted data involved details regarding the study design, the level of masking (open label/single/double), sample size, protocol registration details, study name/ acronym, interventions and comparators, the primary outcomes, the event rates in each arm, the geographical origin of the trial, the randomisation methods used, the level of prevention (primary/secondary) and the methods used to assess intervention adherence. As far as time-to-event outcomes are concerned, extracted data involved the total number of events in each arm over the entire follow-up period of each trial.

\section{Risk of bias}

The risk of bias (RoB) of the selected RCTs was evaluated using the Cochrane RoB V.2.0 tool $^{30}$ by two independent researchers (MPN and KG). Disagreements were resolved via discussion and whenever needed, through the intervention of more experienced researchers (DGG, MGG and DPB).

\section{Calculation of the FI and RFI}

The FI was developed as a measure of RCT robustness. It describes the minimum number of patients within the group with the fewest event count needed to change from 
a non-event to an event, to transform a significant result to a non-significant one. ${ }^{27}$ It is considered as the measurement of the event count, on which the statistical significance depends. $^{22}$

For the current analysis, two researchers (XT and MGG) calculated the FI of each RCT, according to Walsh $e t a l^{27}$ In further detail, after extracting the number of events and nonevents for each trial arm in $2 \times 2$ tables, the additional number of events required to be added in the group with the smaller number of events to make the $p$ value of the Fisher's exact test $\geq 0.05$ was calculated.

An FI equal to zero describes a highly fragile RCT, as zero participants are required to change from a non-event to an event to reverse a significant finding to a non-significant one. $^{22}$

On the other hand, in non-significant comparisons (with an FI equal to 0), the RFI was calculated. This was performed via the subtraction of events from the arm with the fewer events, while simultaneously adding non-events to the same arm, keeping the number of total participants constant, until the Fisher exact test two-sided $P$-value became $<0.05$. Lower RFIs indicate reduced statistical robustness and increased vulnerability to change from statistical non-significance to significance, with only a minimum number of events. At the moment, there is no recognised cut-off for categorising either the FI or the RFI. ${ }^{26}$

For the current analyses, $2 \times 2$ tables were created in Microsoft Excel and the Fischer's exact test was used to calculate and verify the FIs and RFIs of the included trials. For one trial, ${ }^{31}{ }^{32}$ the reported sample and events in each group were used to calculate the FI, and for another, ${ }^{33}$ the incidence and the total number of participants allocated in each group were applied in the FI calculations. When more than two interventions were included in one trial, like in the PREDIMED, each arm was compared with the control diet independently, and the FI or RFI was calculated accordingly, for each paired comparison. When the primary outcome was not reported, the first result presented in the abstract was considered as the primary outcome. In RCTs reporting more than one dichotomous primary outcomes, the FI of all three endpoints was calculated accordingly.

\section{Statistical analyses}

As the research question was 'broad', incorporating all RCTs with MD interventions, an effort to assess differences in RCTs with different characteristics was also performed. Three researchers (KG, MPN and MGG) stratified the selected trials according to blinding, outcome category, sample size, the use of a composite outcome (yes/no) and the method used to assess compliance to the assigned dietary scheme. These categories were used to detect differences in the FI and the RFI between RCTs with different design characteristics and outcomes. As most data did not follow the normal distribution hypothesis, results were presented as medians with their respective IQRs. Group differences were assessed with the Mann-Whitney U test (for comparisons involving two groups) and the Kruskal-Wallis test (for comparisons involving more than two groups). For these analyses, the

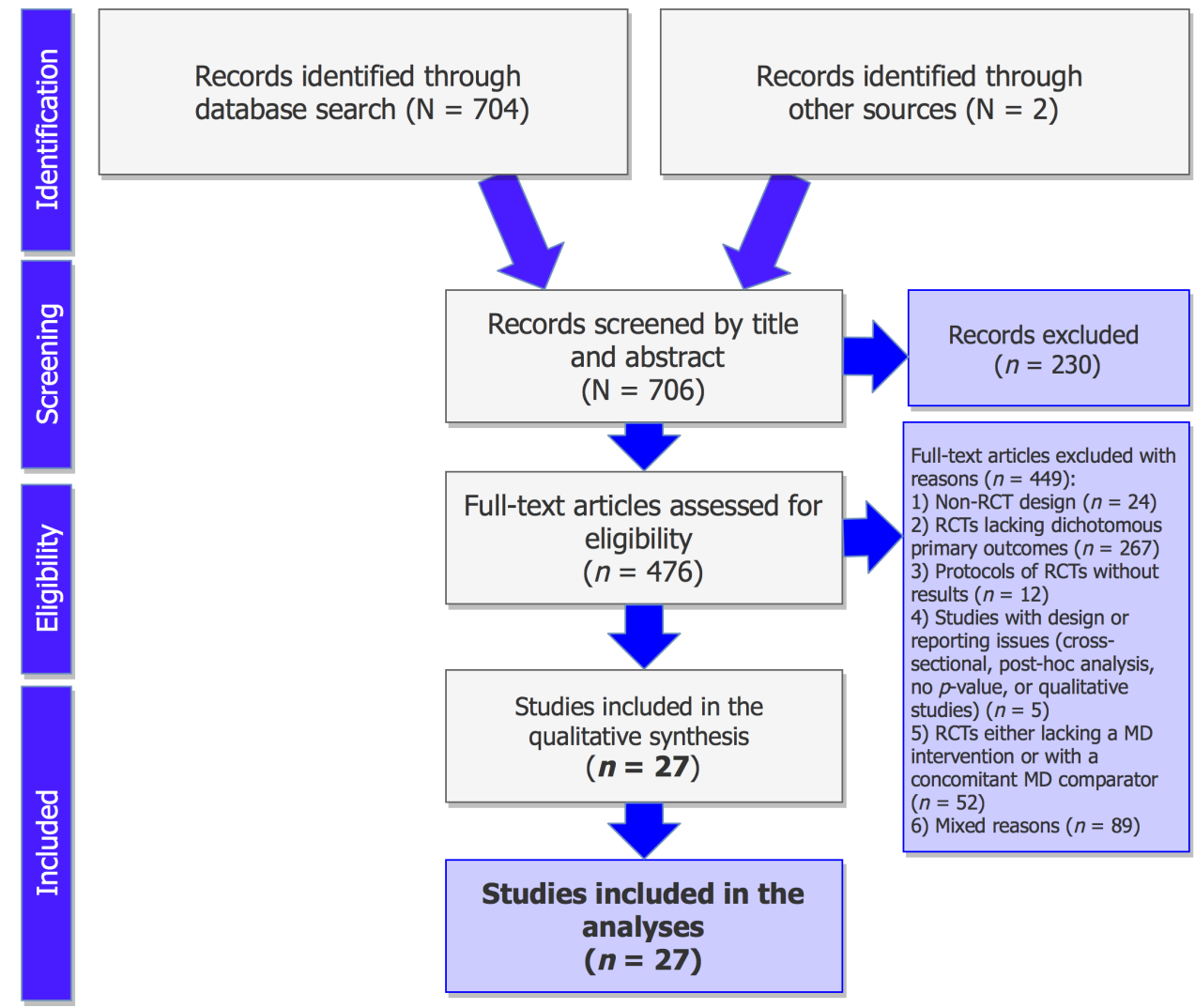

Figure 1 Preferred Reporting Items for Systematic Reviews and Meta-Analyses flow chart of the selection of the studies. MD, Mediterranean diet; RCT, randomised controlled trial. 
Jamovi project (V.0.9.5.16) was used. Significance was set at 0.05 , unless otherwise specified.

\section{RESULTS}

\section{Search results and RCT characteristics}

The detailed process of the selection of RCTs fulfilling the study's criteria is illustrated in figure 1. Published protocols of RCTs lacking the reporting of results, published studies with design issues (cross-sectional, qualitative, or post-hoc analyses), RCTs without dichotomous primary outcomes, and trials lacking a MD intervention, or those with a concomitant MD comparator arm were excluded from the records. A total of 35 distinct publications ${ }^{18} 1931-63$ of parallel interventions were identified meeting the predefined criteria (table 2), with those having an original publication and an erratum being counted as one record (five cases in total). ${ }^{18} 19$ 37-39 44 46-48 63 Multiple publications deriving from the same trials, using the same sample size and outcomes, were also counted as one record (three cases in total). ${ }^{31-33} 353653$ This resulted in 27 distinct RCTs in total, fulfilling the study's criteria and being included in the present analyses.

The majority of RCTs were performed in Spain, ${ }^{18} 1933$ 37-44 46-48 50 52-56 59-61 63 four originated from France $^{34-36} 62$ two took place in the $\mathrm{UK}^{51} 58$ and Italy, ${ }^{31} 32$ and single trials were performed in Australia, ${ }^{45}$ Israe $^{57}$ and India. ${ }^{49}$ Most publications belonged to the PREDIMED or PREDIMED Reus trials, ${ }^{18} 1933$ 37-40 43 44 46-48 50 52-56 61 63 and few referred to the Lyon Heart Study. ${ }^{34-3662}$ Two records involved the St. Carlos gestational diabetes mellitus (GDM) prevention RCT, ${ }^{59} 60$ and others were produced from the Effect of Simple, Targeted Diet in Pregnant Women With Metabolic Risk Factors on Pregnancy Outcomes (ESTEEM), ${ }^{58}$ The Heart Institute of Spokane Diet Intervention and Evaluation Trial,${ }^{51}$ Pre Frail $80,{ }^{41}$ Indo-MD Heart Study ${ }^{49}$ or other trials $^{3132424557}$ (table 2). The sample size ranged from $56^{45}$ to $7403^{44}$ participants.

Given the nature of the intervention (diet), most RCTs were of single-blind masking, and the remaining were open labelled. Regarding the PREDIMED trial, the single-blind masking was disputed by some researchers and further verifications were published by the investigators to support the issue.

\section{Intervention and outcomes}

For one trial, ${ }^{41}$ it was difficult to discern the exact primary outcome. For this specific RCT, ${ }^{41}$ the first result presented in the abstract (reversion to robustness) was considered as the primary outcome. Accordingly, given that the Consolidated Standards of Reporting Trials (CONSORT) ${ }^{64}$ guidelines were produced fairly recently, few-mainly older-trials did not have a preregistered protocol, although some had preceding publications detailing the protocol.

The PREDIMED RCTs ${ }^{18} 1933$ 37-40 4344 46-48 50 52-56 6163 evaluated the efficacy of two MD interventions, one with extravirgin olive oil (EVOO) and one with nuts, in a great variety of health outcomes. In further detail, included PREDIMED RCTs involved the prevention the development of diabetic retinopathy and nephropathy, ${ }^{37}{ }^{38} \mathrm{CVD},{ }^{18} 19$ incidence and reversion of the metabolic syndrome,${ }^{5561}$ liver steatosis, ${ }^{52}$ depression, ${ }^{56}$ osteoporosis-related fractures, ${ }^{33} 53$ peripheral artery disease, ${ }^{54}$ the occurrence of cataract surgery, ${ }^{40}$ as well as the incidence of type 2 diabetes mellitus (T2DM) ${ }^{46-48}$ atrial fibrillation, ${ }^{43}$ breast cancer ${ }^{50}$ and heart failure. ${ }^{44}$ Among the remaining trials, the majority ${ }^{34-364249515762}$ investigated the effects of the MD on CVD risk factors. The St. Carlos GDM prevention ${ }^{5960}$ and the ESTEEM ${ }^{58}$ trials used a MD with EVOO and pistachios to investigate maternal and fetal outcomes. The Pre Frail $80^{41}$ and Properzi ${ }^{45}$ trials applied the MD to evaluate frailty ${ }^{65}$ and non-alcoholic fatty liver (NAFLD) parameters, respectively.

Compliance to the dietary interventions was assessed by the majority of trials using the MEDAS questionnaire, ${ }^{66}$ food frequency questionnaires (FFQs) ${ }^{34-365758}$ including the ESTEEM-Q ${ }^{67}$ previous day 24 hours diet recalls,${ }^{34-3649}$ diet diaries, ${ }^{313251}$ diethistory, ${ }^{45}$ diet 'surveys ${ }^{62}$ or otherscreeners. ${ }^{4258}$ In addition, biomarkers indicative of increased MD adherence were selectively assessed, including urine hydroxytyrosol concentrations and plasma $\alpha$-linolenic acid proportions. When adherence to the control diet differed from that of the intervention group, either a 9-item dietary screener was used, or compliance assessment was not reported in the procedures at all.

\section{Risk of bias}

A summary of the RoB of the included RCTs is presented in figure 2. For some of the PREDIMED RCTs, ${ }^{18} 37444647$ the deviations from the randomisation protocol were considered when assessing the domains of random-sequence generation and allocation-sequence concealment. Many of the PREDIMED RCTs ${ }^{18} 19$ 37-39 44 46-48 63 published errata and reanalyses of their datasets, excluding participants who had deviated from the randomisation protocol; for these, the allocation sequence concealment was considered as adequate, without, however, altering the random-sequence generation domain of the RoB tool, which remained biased. Furthermore, the use of different tools to assess compliance between intervention and controls was also accounted for when assessing the $\mathrm{RoB}$, as it confuted the single-blind masking.

According to the RoB (figure 2), the majority of RCTs exhibited either unclear, or high overall bias. ${ }^{18} 1933353638-424647505254-5759-61$ The fewest concerns were raised with regard to missing outcome data. Among all included RCTs, the ESTEEM ${ }^{58}$ demonstrated the lowest bias throughout the examined RoB domains.

\section{$\mathrm{FI}$ and RFI of the included RCTs}

Table 3 details the FI and RFI of all included RCTs. The majority of comparisons ${ }^{33} 37-4043-4850-5255575861$ failed to provide a significant result between MD intervention and comparator arms, exhibiting an FI equal to 0 . On the other hand, the FI of significant comparisons ranged between $1^{52}$ and $39 .{ }^{60}$ The median FI of the RCTs, excluding those with non-significant comparisons, was 5 . More than half of the comparisons had an FI $<5$, indicating that the addition of 1-4 events to the opposite treatment arm 


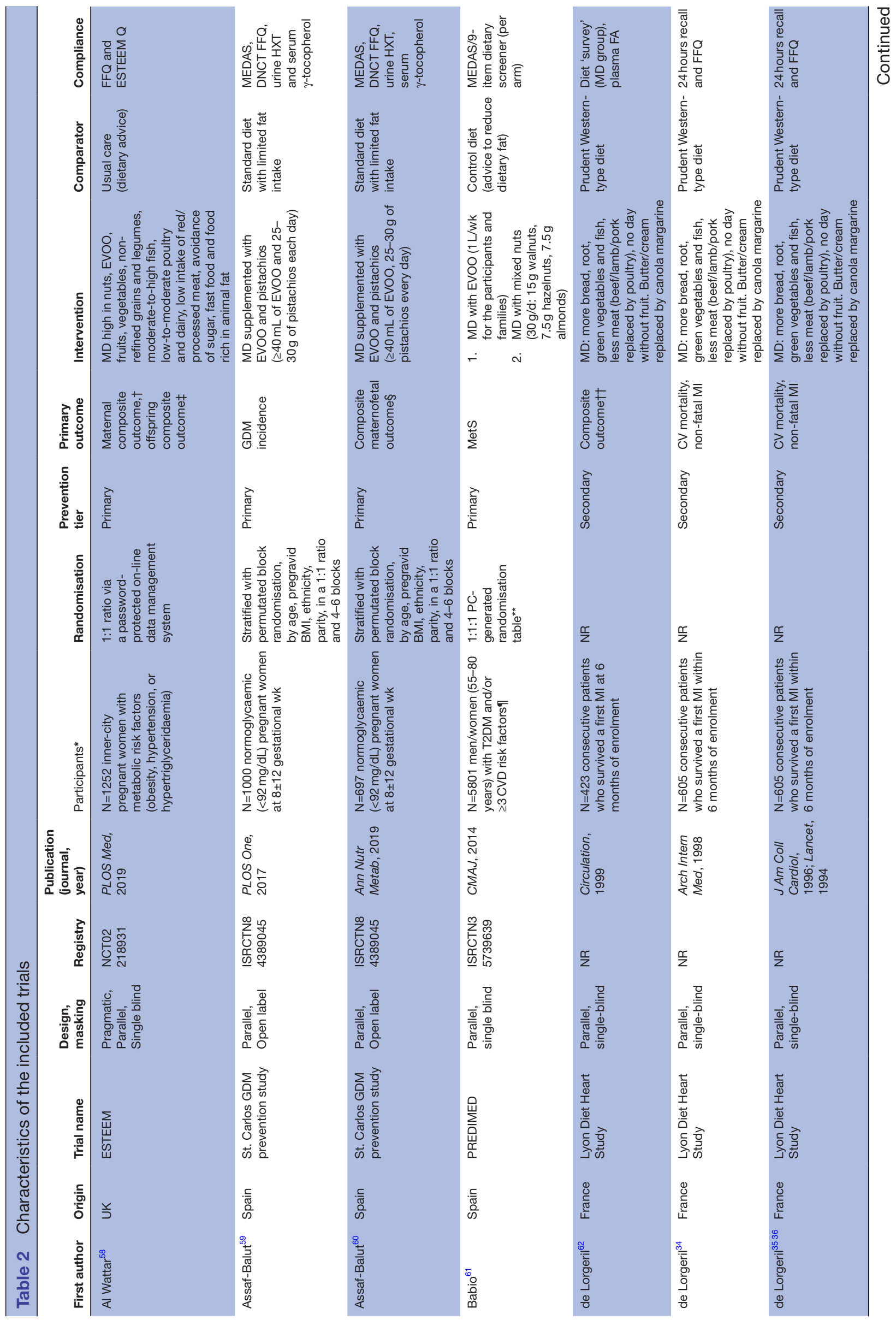




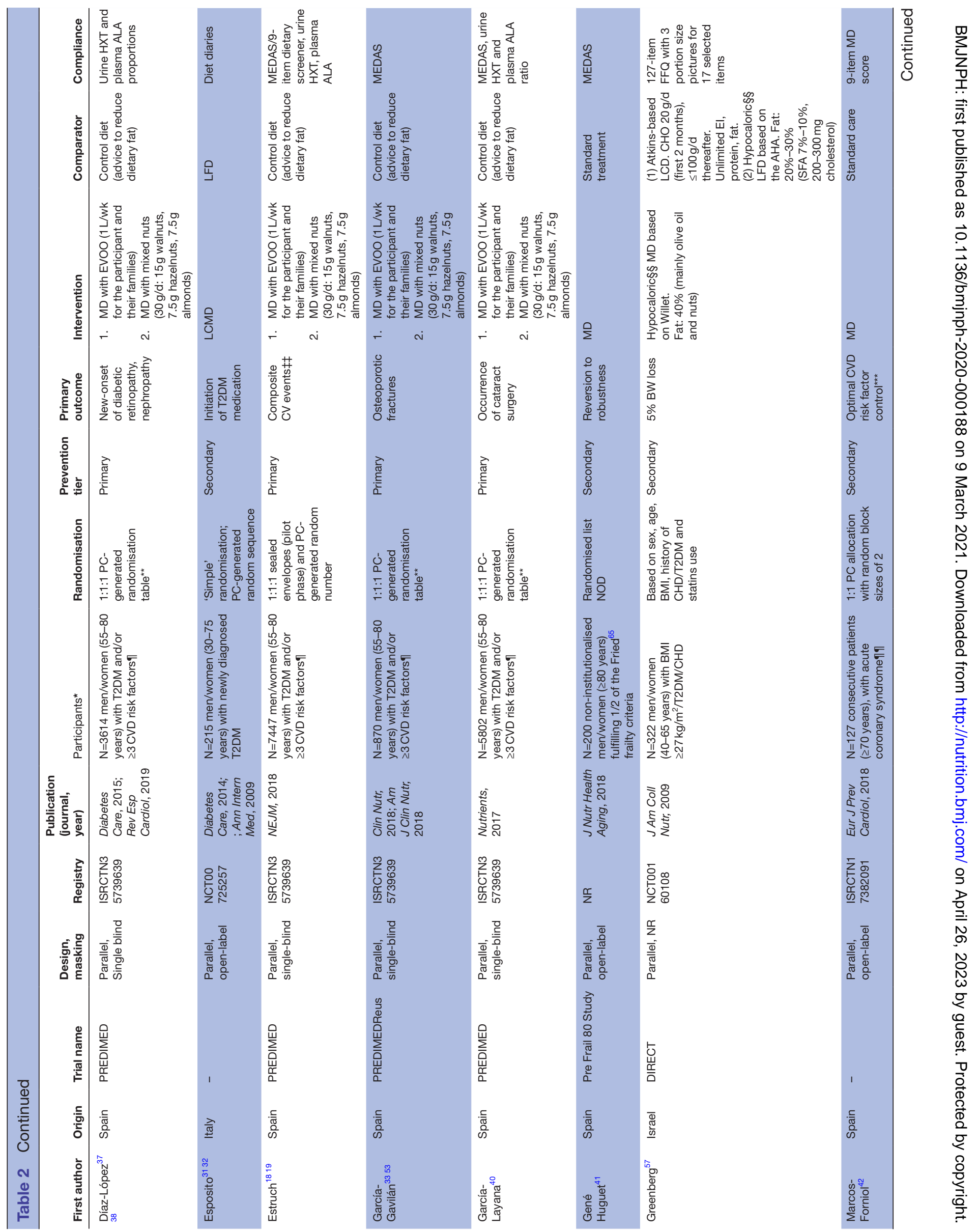




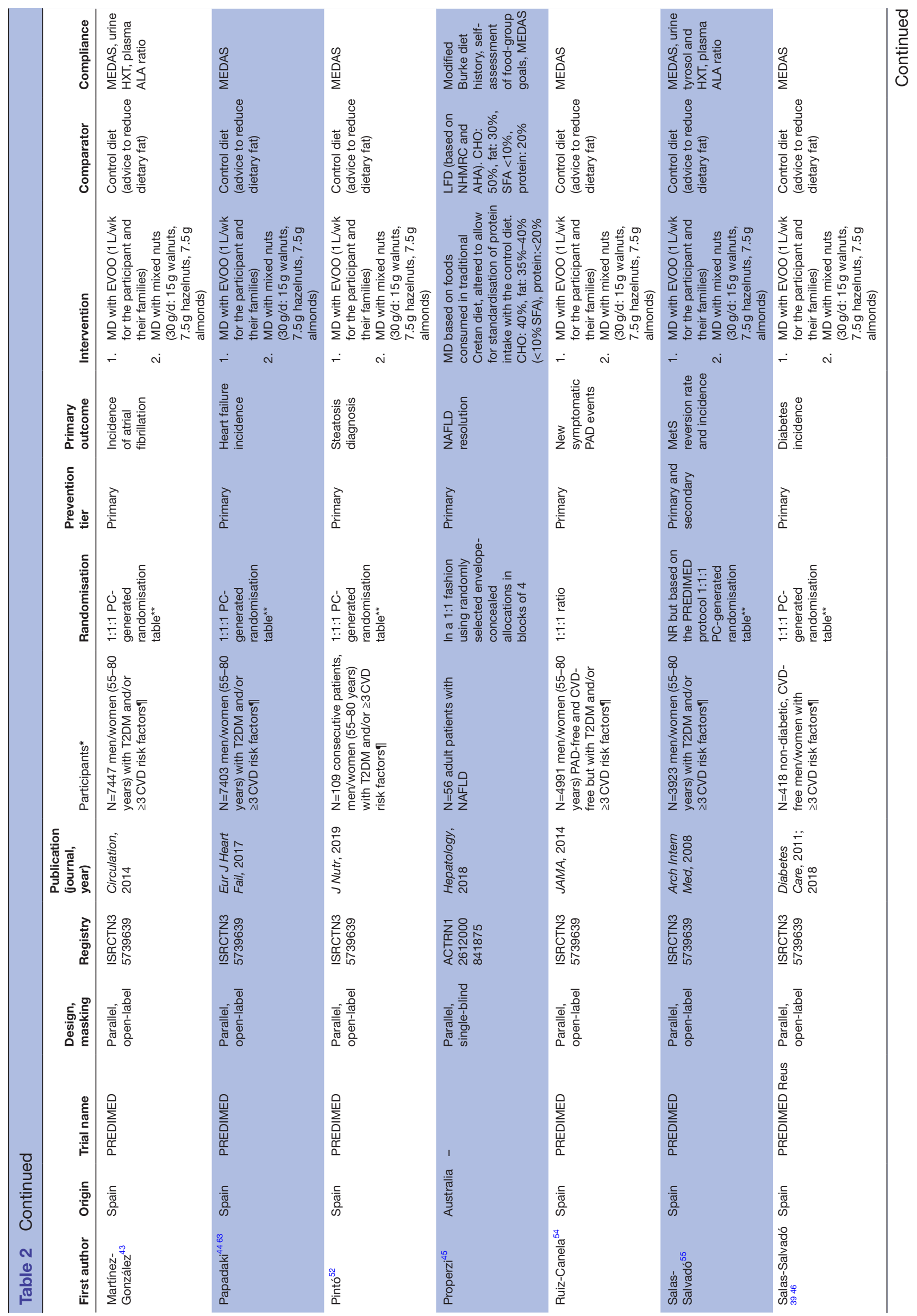

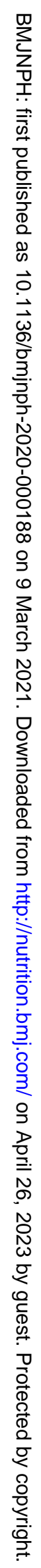



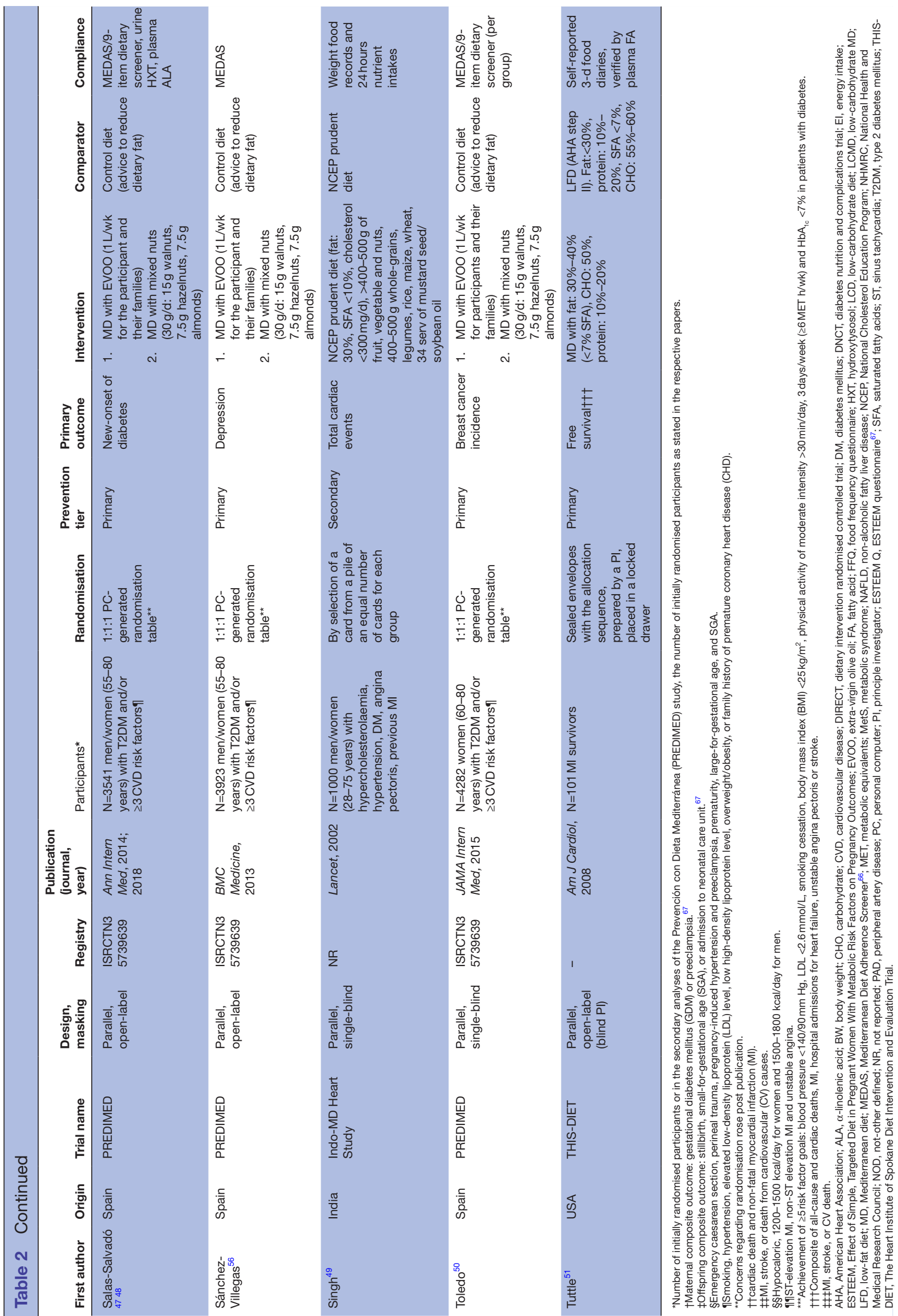


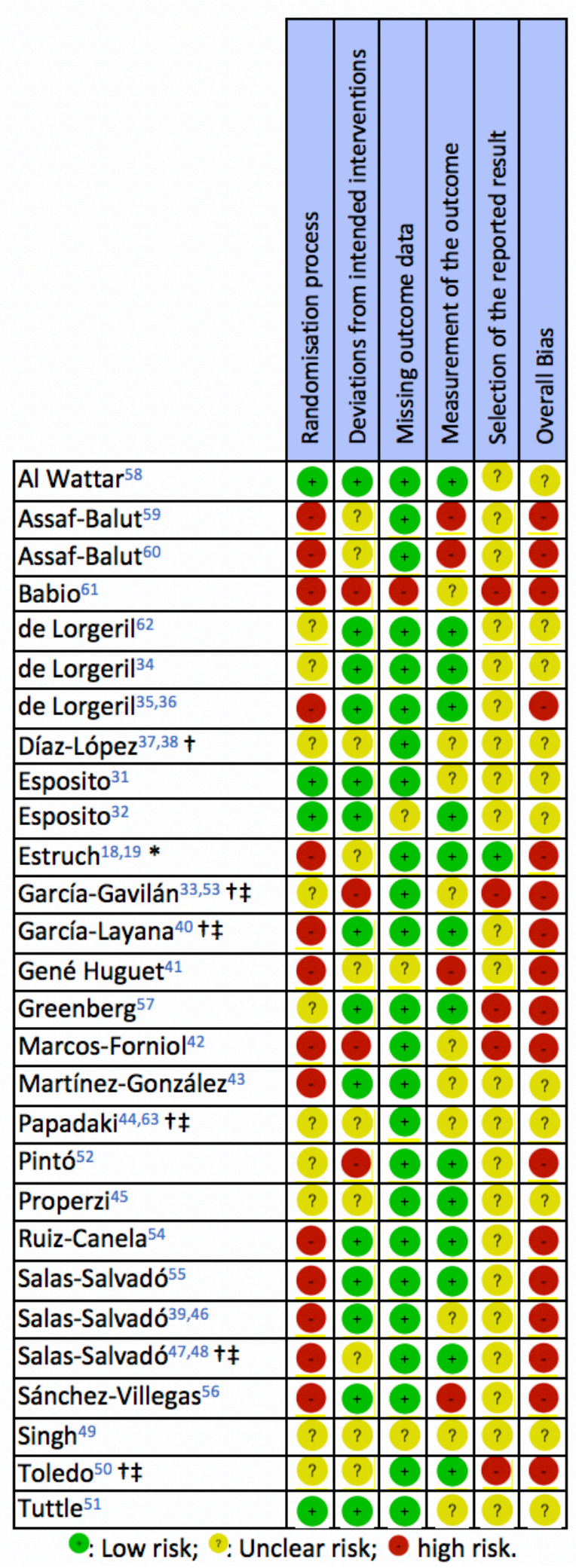

Figure 2 Included randomised controlled trials, investigating the effects of the Mediterranean diet interventions, rated against the Cochrane risk of bias 2.0 tool. ${ }^{30}{ }^{*}$ Publication excluding participants who had deviated from the randomisation protocol. †Concerns regarding randomisation rose post publication. ‡Personnel blinding was reported; however, compliance assessment indicates inadequate blinding of the intervention personnel. eliminated the statistical significance of the RCTs. The most robust results $(\mathrm{FI}>15)$ involved publications of the St. Carlos GDM trial, ${ }^{60}$ the Indo-MD Heart Study, ${ }^{49}$ the Lyon Heart Study ${ }^{62}$ and the PREDIMED comparison between MD + EVOO versus control diet, published by Babio et $a l^{61}$

For the comparisons with an $\mathrm{FI}=0$, the RFI was calculated, ranging between $1^{37-3945} 46$ and $29^{40}$ (median RFI: 7). Six out of 23 comparisons had RFI $<5$ (median: 4), indicating that the change of $1-4$ non-events to events reverses the respective comparisons to statistically significant ones.

\section{Categorisation of the $\mathrm{FI}$ and RFI according to study characteristics}

Table 4 details the FI and RFI categorisation according to the RCT design, the number of participants, and the primary outcome. When masking was accounted for, no differences were noted in the FI or RFI between trials of different allocation masking.

Primary outcomes of the trials were categorised as perinatal, those related to diabetes mellitus or metabolic syndrome, cardiovascular, NAFLD-outcomes, or other (first incidence of breast cancer, cataract surgery, osteoporotic fractures, return to robustness or depression). This allocation failed to induce differences in the FI and RFI between different outcome categories. Similarly, allocation of the trials to those with composite primary endpoints against all others failed to show differences in the FI and RFI between the two groups.

Again, when sample size and methods used to assess dietary compliance between trials were used to allocate the RCTs, no differences were observed in the FI and RFI, with the exception of the RFI among distinct compliancemethods groups $(\mathrm{p} \leq 0.035)$.

\section{DISCUSSION}

The present study revealed that most individual comparisons of RCTs with MD interventions and dichotomous primary outcomes as endpoints fail to demonstrate significant results. In parallel, those with comparisons yielding significant findings appear fragile, with a small number of events needed to change the result from significant to non-significant. Subsequently, the number of robust RCTs investigating $\mathrm{MD}$ interventions appears to be limited.

Among the reviewed trials, the St. Carlos $\mathrm{GDM}^{60}$ and the PREDIMED RCT conducted by Babio ${ }^{61}$ exhibited the highest FIs, indicating that nutrition RCTs can be robust. Both of these trials exhibited high RoB in several RoB domains, suggesting that robustness does not necessarily coincide with low RoB. In the St. Carlos GDM study, ${ }^{60}$ the reported event rate was high, corresponding to $27.8 \%$ and $25.8 \%$ of the intervention and control groups, respectively, whereas the Babio ${ }^{61}$ PREDIMED trial did not exhibit a similar high rate of events $(1.5 \%$ of the total participants in the intervention arm and $3.9 \%$ of those allocated in the control group, respectively). This 


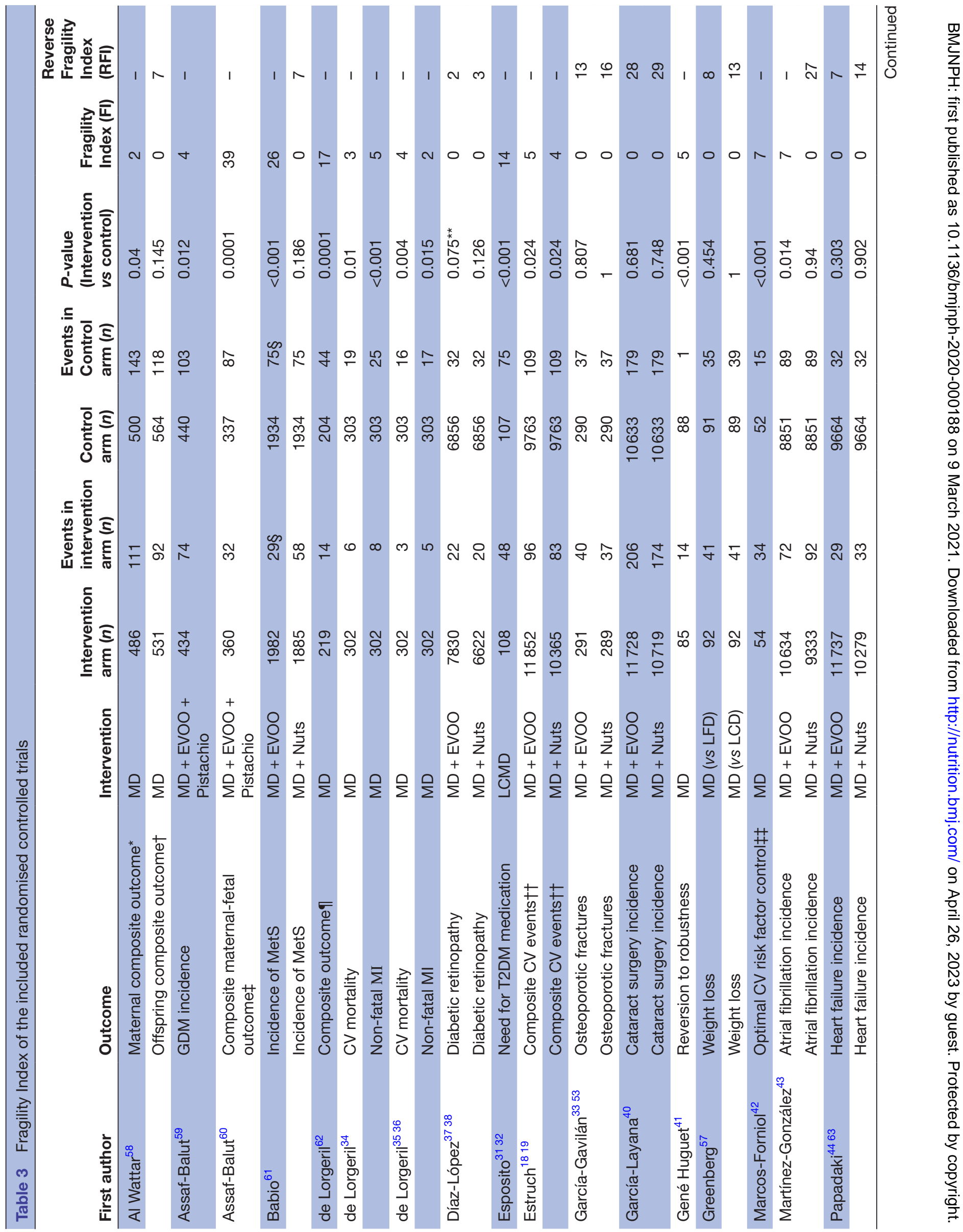




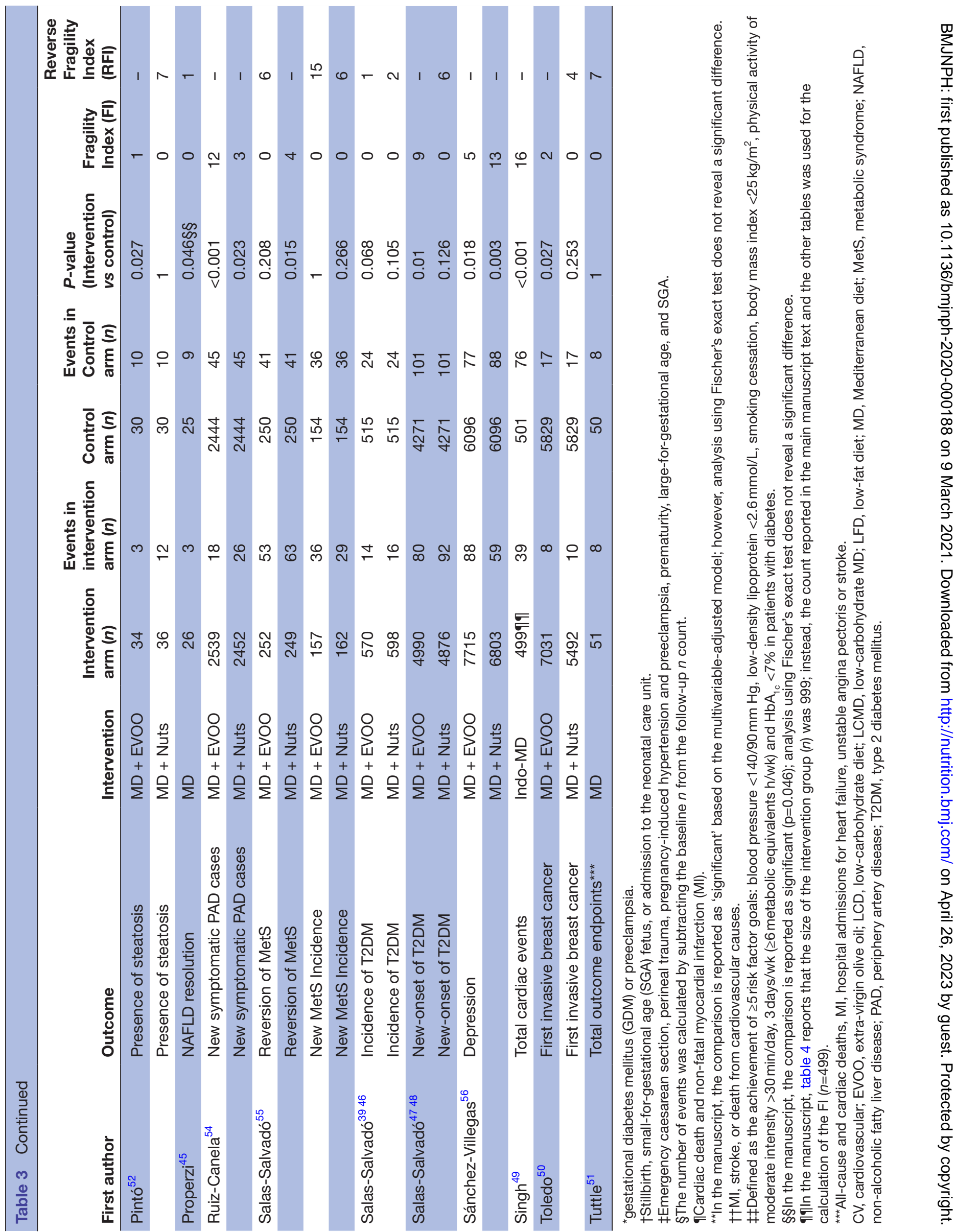


Table 4 Categorisation of the Fragility and Reverse Fragility Index according to randomised controlled trial design, sample size, compliance assessment method and primary outcomes ( $n$, median and IQR)

\begin{tabular}{|c|c|c|c|c|c|}
\hline & $\mathbf{N}$ & Fragility Index & $P$-value & Reverse Fragility Index & $P$-value \\
\hline \multicolumn{6}{|l|}{ Masking } \\
\hline Single blind & 21 & $4.0(2.5-10.5)$ & $0.38^{*}$ & $7.0(3.3-15.3)$ & $0.68^{*}$ \\
\hline Not reported & 2 & $\mathrm{CNC}$ & & $10.5(0.3-11.8)$ & \\
\hline \multicolumn{6}{|l|}{ Outcome categorisation } \\
\hline Cardiovascular outcomes & 16 & $5.0(3.8-8.3)$ & & $10.5(7.0-17.3)$ & \\
\hline Outcomes related to NAFLD & 3 & $1.0(1.0-1.0)$ & & $4.0(2.5-5.5)$ & \\
\hline Othert & 9 & $5.0(4.3-7.0)$ & & $16.0(13.0-28.0)$ & \\
\hline \multicolumn{6}{|l|}{ Composite outcome } \\
\hline$<1000$ patients & 20 & $5.0(4.0-7.0)$ & $0.64 \S$ & $7.0(2.0-13.0)$ & $0.57 \S$ \\
\hline$\geq 1000$ patients & 27 & $7.0(3.5-12.5)$ & & $7.0(6.0-14.8)$ & \\
\hline \multicolumn{6}{|l|}{ Dietary compliance assessment methods } \\
\hline Questionnaires (FFQs, diet scores)/diet history & 22 & $5.0(2.3-10.8)$ & $0.59^{*}$ & $7.0(6.3-13.0)$ & $0.035^{\star}$ \\
\hline Diet recalls or records & 7 & $4.5(3.3-11.8)$ & & $2.0(1.5-2.5)$ & \\
\hline Biomarkers & 18 & $6.0(4.0-11.0)$ & & $11.0(6.0-27.3)$ & \\
\hline
\end{tabular}

\section{*Based on the Kruskal-Wallis test.}

†First incidence of breast cancer, cataract surgery, osteoporotic fractures, return to robustness, depression.

‡Maternal/offspring composite outcome, incidence of metabolic syndrome (MetS), cardiovascular (CV) mortality, composite CV events, optimal CV risk factor control, total cardiac events, total outcome endpoints.

$\S$ Based on the Mann-Whitney U test.

Пincidence of gestational diabetes mellitus (GDM), atrial fibrillation, heart failure, type 2 diabetes mellitus (T2DM), depression, first invasive breast cancer, steatosis, non-fatal myocardial infarction, cataract surgery, diabetic retinopathy, osteoporotic fractures, need for T2DM medication, reversion to robustness, weight loss, non-alcoholic fatty liver disease (NAFLD) resolution, new symptomatic periphery artery disease cases, reversion of MetS.

$\mathrm{CNC}$, could not be calculated; DM, diabetes mellitus; FFQ, food frequency questionnaire; ;IQR, interquartile range.

discrepancy between two RCTs with high FI indicates that the event rate is not the only parameter influencing the FI. According to Gaudino $e t a l^{99}$ the FI, $P$-values, events and sample size are mathematically related; however, the type of primary outcome might also have an effect on a trial's robustness. For instance, the St. Carlos GDM study ${ }^{60}$ used two primary outcomes, the first being the incidence of $\mathrm{GDM}^{59}$ and the second being a composite maternal-fetal score $^{60}$ and published the trial's results in two distinct publications. Although both publications reported significant findings, the first exhibited an FI equal to $4^{59}$ and the latter an FI of $39 .{ }^{60}$ Composite scores are popular in nutrition research; they are combining distinct outcomes, often resulting in a greater event rate as compared with the use of the 'component' outcomes independently. In the present analyses, the use of composite scores did not ensure statistical robustness in all of the trials herein, with many exhibiting low FIs and RFIs $(<5) .{ }^{18} 19353658$

The Esposito et $a l^{3132}$ trial also demonstrated a high FI, indicating a robust outcome. However, in this specific RCT the two diets applied by the trialists were not so comparable. In more detail, the intervention arm adopted a low-calorie MD, whereas the comparator group followed a low-fat diet, without any reported restrictions concerning the energy intake. Thus, the observed effects of the intervention arm, and subsequently the high FI, could well have been the result of the prescribed lowcalorie diet, as restricted energy intake leading to weight loss has been shown to delay the development of T2DM and subsequently, improve glycaemic control and various coronary factors. ${ }^{68-72}$

Additionally, it appears that the majority of evidence on MD interventions with dichotomous outcomes is based on the PREDIMED trial, which had a multiarm design. According to Parmar et al, ${ }^{73}$ trials concurrently evaluating more than one intervention, like the PREDIMED, have increased chances of finding significant differences even with the use of small sample sizes. Since the FI is based on Fischer's exact test it can only be applied on $2 \times 2$ tables, thus in trials with three parallel arms, distinct comparisons of each intervention with the comparator group were performed for the calculation of the FI/RFI. Exlcusion of 
the trials with three arms however, did not alter the pooled results herein (Median FI: 5, RFI: 7). Accordingly, separation of the PREDIMED comparisons revealed a lack of a significant effect in approximately half of the comparison pairs. When the PREDIMED comparison arms where grouped together and compared against the other trials, the median FIs and RFIs between groups were similar (5 and 7, respectively for both groups), indicating a similar robustness to the rest of MD RCTs. Apart from disputes concerning the randomisation of the PREDIMED sample and the different reported tools used to assess compliance, Correia ${ }^{74}$ also noted discrepancies in the medical care offered to the participants, resulting in allocation concealment bias. In parallel, the control group received an intervention of lower intensity for the initial 3 years of the RCT, a corrected problem before completion of the recruitment and analysis of the results. ${ }^{75}$ Inevitably, however, a different intervention frequency unmasks participant allocation. Additionally, compliance with the low-fat control diet appeared to be a difficult task in the long run, with the mean fat intake of participants reaching $37.4 \%$ of the total energy intake 5 years post intervention. Thus, the control diet did not correspond to a low-fat regime but was rather lower in the fat content compared with the two MD interventions ( $42 \%$ of the total energy intake) ${ }^{75}$ Subsequently, more losses to follow-up were recorded in controls, mainly among participants with a worse CVD risk profile at recruitment. ${ }^{75}$ This induced further bias towards ameliorated results for the control group, leading to mitigated between-groups differences, and by inference, the bias in the FI. Despite the issues mentioned above, the PREDIMED is an ambitious milestone trial for nutrition research and reanalysis of the data did not reveal differences in the reported results despite the randomisation issues. Given the prolonged intervention duration and the large number of participants, collaborators and outcomes, it is not uncanny that certain aspects of the trial's design and execution demonstrated issues. Undoubtedly, similar issues might have also been observed in pharmacological trials. On a sidenote, the PREDIMED is probably the only megatrial that has undergone this degree of exhaustive scrutiny, despite the results being unchanged at republication. Moreover, unlike pharmacological trials, the trial aimed in providing evidence to a more traditional and accessible therapy (i.e. diet), without supporting any industry products other than common, 'healthy' foods, including olive oil and nuts. According to the authors, these issues should have increased trust to the results. However, for the detailed methodologists, the majority of nutrition research has limitations, whereas for the sceptics, nutrition research is scrutinised for competing against the big Pharma on a pretence of evidence.

For many of the included trials, the calculated low FIs and RFIs were associated with an overall smaller number of events. This problem can be surpassed if greater sample sizes are recruited at baseline, or if we shift the focus towards the execution of pragmatic trials. However,
Gaudino $^{29}$ noted that it is more ethical to power RCTs in order to produce the required level of evidence using the minimum possible number of participants. Enrolling additional participants might result in stronger evidence against the null hypothesis, however, it might violate the equipoise principle. ${ }^{29}$ On the other hand, findings may produce more contradictory results than similar trials, and may also pose further ethical concerns. ${ }^{29}$

An important question arising from the present findings is whether we are receiving the reliable data we are craving for, by performing RCTs, or if we are overlooking important flaws of either the nutrition science, or the methodology applied in trials examining the MD. However, the present study did not aim in examining the importance or the effectiveness of the MD as a therapeutic dietary regimen. The low robustness calculated herein indicates that even the best level of primary MD evidence proving causality, namely the RCTs, can fail to reach the standards one would expect. Recently, a study ${ }^{76}$ assessing the FI of clinical nutrition trials revealed a low FI. According to Zeilstra, ${ }^{77}$ many nutritional RCTs yield ambiguous results, which is why the RCT design is often considered 'ill-suited' for nutritional research. ${ }^{77-80}$ Additionally, given that most trials are based on different analyses of the same landmark protocol (PREDIMED), bias and limitations of the trial are inevitably reproduced in every publication. Subsequently, any synthesis of related RCTs, although it may present low heterogeneity, carries an inherited risk of extrapolated findings. To nutrition's defence however, lower median FI compared with that of MD interventions has been reported in perioperative, ${ }^{81}$ anesthesiology, ${ }^{82}$ plastic surgery, ${ }^{83}$ and critical-care medicine $^{84}$ RCTs, as well as among paediatric orthopedic ${ }^{85}$ and appendicitis ${ }^{86}$ trials. Nevertheless, the synthesis of these trials for recommendations formulation consists of a common practice in the fields mentioned above, as in the science of nutrition.

On the flip side, RCTs with MD interventions and continuous primary outcomes demonstrate significant findings while supporting the health benefits of adhering to the MD prototype. However, similarly to the Esposito ${ }^{31} 32$ trial, control interventions are not always comparable, with a tendency to favour the MD arms. This is why, to verify the health effects of MD adherence and advocate for its prescription, superiority trials with continuous primary outcomes should be performed, comparing the MD to other healthy diet regimens instead of the usual diet of participants or dietary advice only.

Although the current results indicate that as far as trials with dichotomous outcomes are concerned, the evidence on the MD entails some limitations, several other factors must also be considered before treating the MD with contempt. For instance, assessment of the participants' adherence to the dietary intervention, often relies on short dietary indexes instead of more objective measures, and consists of an important component of a nutrition RCT. Moreover, the Hawthorn effect ${ }^{87}$ (individuals modify an aspect of their behaviour in response 
to their awareness of being observed) is apparent in all of nutrition research; thus, compliance and assessment are not always accurate. RCTs are often used to guide clinical practice and are sometimes incorporated in clinical practice guidelines intact or after synthesis, using systematic reviews and meta-analyses. Given the demand for evidence-based nutrition recommendations, ${ }^{88-91}$ the results suggest that the formulation of recommendations promoting the MD based on RCTs should be performed with caution. ${ }^{76}$ Thorough examination of the American College of Gastroenterology guidelines revealed that most RCTs used to guide recommendations regarding Crohn's disease relied on a small number of superior events for 'securing' statistical significance. ${ }^{92}$ Often, the FI coincided with the drop-outs reported in some trials. This is why, reporting the FI has also been suggested for systematic reviews and meta-analyses, to understand the fragility of the presented associations and identify possible misuse of the $P$-value. ${ }^{93}$ The present study aimed to pinpoint another issue requiring the attention of scientists when performing nutrition trials, namely the FI. Meticulous care in the trial design, sample size and execution can improve the FI of nutrition trials and aid in upgrading the science of nutrition, as succinctly pointed out by other researchers. ${ }^{94}$

Another important issue in nutrition research is that often, detailed definitions of the interventions are not reported. This is also the case with the MD. Although the label MD is a generic term used to describe the diet of inhabitants around the Mediterranean basin, according to Trichopoulou, ${ }^{95}$ what constitutes the MD and its key determinants differs even among 'experts' worldwide. Martínez-González ${ }^{96}$ noted that the discrepancies in the MD definition consist of a major problem, especially for intervention studies. As a result, except for the RCTs included herein which were stemming from the same protocol, like the PREDIMED, the remaining trials have most probably used different definitions of the MD. For instance, Singh and associates ${ }^{49}$ used a National Cholesterol Education Program modification of the MD, whereas Greenberg et a $\tilde{l}^{\tilde{7}}$ reported following Professor Willet's definition of the MD. This indicates that differences may exist even under the same intervention label, and these may well induce inconsistencies and bias in the reported outcomes. ${ }^{97}$

Undoubtedly, one important limitation of the study stems from the relatively small number of RCTs with a dichotomous primary outcome included in the analyses. However, one should consider that the total number of RCTs examining MD interventions is rather small; additionally, in the present study, RCTs were selected based on a systematic search strategy; thus, the results reflect the actual number of available MD-RCTs fulfilling the study's criteria and being indexed in the PubMed database. An additional limitation is that the publication of many RCTs predated the CONSORT ${ }^{64} 98$ guidelines; thus, few important characteristics have not been reported. In parallel, in the case of MD RCTs, as in the majority of nutritional epidemiology, diet adherence and intake rely on not so precise exposure assessments-mainly selfreported information-with an increased potential for confounding. ${ }^{169-101}$

Moreover, due to the small number of retrieved trials, it was not possible to correlate the FI with individual study characteristics, or to perform additional statistical analyses. As already mentioned, the use of broad research topics for the assessment of the FI/ RFI, as seen herein with the MD, is common in the literature. ${ }^{26}{ }^{76}$ Although such studies result in pooling a greater number of RCTs, they also tend to mix many studies with non-comparable aspects, including participant age, health status, study question, outcomes categories, etc. In an effort to correct the heterogeneity observed in the included trials, we also calculated the FI and the RFI after allocating the RCTs based on sample sizes, masking, or outcomes categories. However, these analyses failed to reveal differences, with the only observed significant finding involving the different RFI among RCTs using different methods to assess dietary adherence. Therefore, in the pooled sample of RCTs included herein, differences in sample size, outcomes categories or masking had a minimal effect on the FI and the RFI. Nevertheless, a larger pool of RCTs might have produced different results.

Limitations of the FI include the fact that its calculation is based on the Fischer's exact test, which is considered as stricter and more prone to type II errors when compared with the $\chi^{2}$ test. Additionally, as already mentioned, it can only be applied to dichotomous outcomes, whereas the majority of nutrition research tends to examine continuous outcomes. Furthermore, the lack of standardised cut-offs for categorising RCTs as either robust, or fragile, is evident. ${ }^{102} 103$ According to Andrade, ${ }^{102}$ the most important limitation of the index concerns the use of the much decried statistical threshold $(\mathrm{p}<0.05)$ for determining the significance of a study's outcome. However, one should consider that the FI uses the same threshold applied in the published RCTs and that additionally, the FI is highly correlated to the $P$-value of a trial, with a significance closer to 0.05 indicating a lower FI. ${ }^{103} 104$ Moreover, although Walsh ${ }^{27}$ suggested calculating the index in time-to-event data-as performed in the current analysis-several researchers raised concerns, claiming that it cannot account for the effect of time. ${ }^{102}$ Nevertheless, as Charilaou ${ }^{105}$ promptly noted, the FI can offer a measure of the validity of an RCT, especially in trials where the number of participants lost to follow-up, exceeded the FI of the trial. More recently, in a collective effort to optimise patient care, the routine use of the FI has been recommended for the development of all clinical practice guidelines ${ }^{28}$ with incorporation of the results in the GRADE (Grading of Recommendations Assessment, Development and Evaluation) format. 


\section{CONCLUSIONS}

In summary, the present study reveals that, when adhering to good scientific principles, one discerns that even in the top tiers of evidence hierarchy, research on the MD may lack robustness, setting concerns for the formulation of nutrition recommendations in a wider context. A collective effort is required to promote the science of nutrition in an evidence-based manner. Despite the mediocre robustness of RCTs with MD interventions, the findings herein do not overlay on the importance of the MD on health or as a UNESCO accredited intangible cultural heritage. Nevertheless, it appears that our quest for an ideal diet for all could prove horses for courses, and a more personalised approach may be required for both health attainment and ameliorated disease outcomes. As Correia $^{74}$ noted 'enthusiasm regarding the MD may not be proportional to the level of evidence' and this might lead to allegiance bias and an imbalance between expectancies and evidence.

\section{Author affiliations}

${ }^{1}$ Unit of Reproductive Endocrinology, 1st Department of Obstetrics and Gynecology, Faculty of Health Sciences, Aristotle University of Thessaloniki, Thessaloniki, Central Macedonia, Greece

${ }^{2}$ Rheumatology and Clinical Immunology, Faculty of Health Sciences, University of Thessaly, Larissa, Greece

${ }^{3}$ Nutritional Sciences \& Dietetics, Faculty of Health Sciences, International Hellenic University, Thessaloniki, Greece

${ }^{4}$ Medical School, Faculty of Health Sciences, Aristotle University of Thessaloniki, Thessaloniki, Central Macedonia, Greece

${ }^{5}$ Department of Endocrinology, Diabetes and Metabolism, Hippokration General Hospital of Thessaloniki, Thessaloniki, Central Macedonia, Greece

${ }^{6}$ Laboratory of Histology and Embryology, Medical School, Faculty of Health Sciences, Aristotle University of Thessaloniki, Thessaloniki, Central Macedonia, Greece

${ }^{7}$ Division of Transplantation, Immunology and Mucosal Biology, MRC Centre for Transplantation, School of Medical Education, King's College London, London, UK

Twitter Meletios P Nigdelis @melnigdelis, Xenophon Theodoridis @x_theodoridis and Dimitrios P Bogdanos @dbogdanos

Acknowledgements The present work has been submitted for presentation at the 10th Scientific Congress of the Medical School, Aristotle University of Thessaloniki (2021).

Contributors MGG and DGG developed the research question and finalised the paper inclusion. MPN, MGG, KG and XT performed the search and extracted the data. AT and TP participated in the search. KG designed the protocol. XT, MGG and DPB calculated the FI and the RFI. MPN and KG assessed the risk of bias of the included trials, with some help from MGG and supervision from DGG and DPB. MPN and MGG performed the statistical analyses. KG, MPN, XT, TP and MGG prepared tables and figures. DGG, DPB and MGG interpreted results, drafted and finalised the manuscript. All authors read the manuscript, contributed comments to its revision, and have approved and agreed to the final version. MGG submitted the manuscript, and DGG is responsible for the overall content as guarantor.

Funding The present research was funded by the "MSc in Health and Environmental factors", Medical School, Aristotle University of Thessaloniki.

Competing interests None declared.

Patient consent for publication Not required.

Provenance and peer review Not commissioned; externally peer reviewed.

Data availability statement Although most of the data are already presented in the manuscript text, they are also available upon reasonable request. For expression of interest, please contact Prof. Dimitrios G. Goulis.

Open access This is an open access article distributed in accordance with the Creative Commons Attribution Non Commercial (CC BY-NC 4.0) license, which permits others to distribute, remix, adapt, build upon this work non-commercially, and license their derivative works on different terms, provided the original work is properly cited, appropriate credit is given, any changes made indicated, and the use is non-commercial. See: http://creativecommons.org/licenses/by-nc/4.0/.

\section{ORCID IDs}

Maria G Grammatikopoulou http://orcid.org/0000-0003-4167-6595

Meletios P Nigdelis http://orcid.org/0000-0003-3865-6833

Xenophon Theodoridis http://orcid.org/0000-0001-9810-7583

Konstantinos Gkiouras http://orcid.org/0000-0002-6407-8494

Antigoni Tranidou http://orcid.org/0000-0001-6406-8424

Theodora Papamitsou http://orcid.org/0000-0002-0709-5407

Dimitrios P Bogdanos http://orcid.org/0000-0002-9697-7902

Dimitrios G Goulis http://orcid.org/0000-0002-5005-1995

\section{REFERENCES}

1 Keys A, Menotti A, Karvonen MJ, et al. The diet and 15-year death rate in the seven countries study. Am J Epidemiol 1986;124:903-15.

2 Minelli P, Montinari MR. The Mediterranean diet and cardioprotection: historical overview and current research. $J$ Multidiscip Healthc 2019;12:805-15

3 Grammatikopoulou MG, Gkiouras K, Tranidou A, et al. Food security and adherence to the Mediterranean diet: an interplay of socio-demographic characteristics. In: Preedy VR, Watson RR, eds. The Mediterranean Diet: An Evidence-Based Approach. 2nd edn. London, U.K: Elsevier, 2020: 79-88.

4 Rees K, Takeda A, Martin N, et al. Mediterranean-style diet for the primary and secondary prevention of cardiovascular disease. Cochrane Database Syst Rev 2019;3:CD009825.

5 Esposito K, Maiorino MI, Bellastella G, et al. A journey into a Mediterranean diet and type 2 diabetes: a systematic review with meta-analyses. BMJ Open 2015;5:e008222.

6 Becerra-Tomás N, Blanco Mejía S, Viguiliouk E, et al. Mediterranean diet, cardiovascular disease and mortality in diabetes: a systematic review and meta-analysis of prospective cohort studies and randomized clinical trials. Crit Rev Food Sci Nutr 2019:1-21.

7 Grosso G, Marventano S, Yang J, et al. A comprehensive metaanalysis on evidence of Mediterranean diet and cardiovascular disease: are individual components equal? Crit Rev Food Sci Nutr 2017;57:3218-32.

8 Amati F, Hassounah S, Swaka A. The impact of Mediterranean dietary patterns during pregnancy on maternal and offspring health Nutrients 2019;11. doi:10.3390/nu11051098. [Epub ahead of print: 17 May 2019].

9 Gonzalez-Campoy JM, St Jeor ST, Castorino K, et al. Clinical practice guidelines for healthy eating for the prevention and treatment of metabolic and endocrine diseases in adults: cosponsored by the American association of clinical Endocrinologists/the American College of endocrinology and the obesity Society. Endocr Pract 2013;19 Suppl 3:1-82.

10 Ivers NM, Jiang M, Alloo J, et al. Diabetes Canada 2018 clinical practice guidelines: key messages for family physicians caring for patients living with type 2 diabetes. Can Fam Physician 2019;65:14-24.

11 Olszewski TM. The causal conundrum: the Diet-Heart debates and the management of uncertainty in American medicine. J Hist Med Allied Sci 2015;70:218-49.

12 Schoenfeld JD, loannidis JPA. Is everything we eat associated with cancer? A systematic cookbook review. Am J Clin Nutr 2013;97:127-34.

13 loannidis JPA. Biases in obesity research: identify, correct, endorse, or abandon effort? Obesity 2016;24:767-8.

14 Trepanowski JF, loannidis JPA. Perspective: limiting dependence on nonrandomized studies and improving randomized trials in human nutrition research: why and how. Adv Nutr 2018;9:367-77.

15 Tatsioni A, Bonitsis NG, loannidis JPA. Persistence of contradicted claims in the literature. JAMA 2007;298:2517.

16 Kristal AR, Peters U, Potter JD. Is it time to abandon the food frequency questionnaire? Cancer Epidemiol Biomarkers Prev 2005;14:2826-8.

17 Oxford Centre for Evidence-based Medicine. Oxford centre for evidence-based medicine - levels of evidence, 2009. Available: https://www.cebm.net/2009/06/oxford-centre-evidence-basedmedicine-levels-evidence-march-2009/

18 Estruch R, Ros E, Salas-Salvadó J, et al. Primary prevention of cardiovascular disease with a Mediterranean diet supplemented with extra-virgin olive oil or nuts. N Engl J Med 2018;378:e34. 
19 Estruch R, Ros E, Salas-Salvadó J, et al. Primary prevention of cardiovascular disease with a Mediterranean diet. N Engl J Med 2013;368:1279-90.

20 Agarwal A, loannidis JPA. PREDIMED trial of Mediterranean diet: retracted, republished, still trusted? BMJ 2019;::1341.

21 loannidis JP. We need more randomized trials in nutrition-preferably large, long-term, and with negative results. Am J Clin Nutr 2016;103:1385-6.

22 Svantesson E, Hamrin Senorski E, Danielsson A, et al. Strength in numbers? the fragility index of studies from the Scandinavian knee ligament registries. Knee Surg Sports Traumatol Arthrosc 2020;28:339-52.

23 It's time to talk about ditching statistical significance. Nature 2019;567:283.

24 Amrhein V, Greenland S, McShane B. Scientists rise up against statistical significance. Nature 2019;567:305-7.

25 McShane BB, Gal D, Gelman A, et al. Abandon statistical significance. Am Stat 2019;73:235-45.

26 Khan MS, Fonarow GC, Friede T, et al. Application of the reverse fragility index to statistically Nonsignificant randomized clinical trial results. JAMA Netw Open 2020;3:e2012469.

27 Walsh M, Srinathan SK, McAuley DF, et al. The statistical significance of randomized controlled trial results is frequently fragile: a case for a fragility index. J Clin Epidemiol 2014;67:622-8.

28 Tignanelli CJ, Napolitano LM. The fragility index in randomized clinical trials as a means of optimizing patient care. JAMA Surg 2019;154:74-9.

29 Gaudino M, Hameed I, Biondi-Zoccai G, et al. Systematic evaluation of the robustness of the evidence supporting current guidelines on myocardial revascularization using the fragility index. Circ Cardiovasc Qual Outcomes 2019;12:e006017.

30 Higgins JPT, Altman DG, Gøtzsche PC, et al. The Cochrane collaboration's tool for assessing risk of bias in randomised trials. BMJ 2011;343:d5928.

31 Esposito K, Maiorino MI, Petrizzo M, et al. The effects of a Mediterranean diet on the need for diabetes drugs and remission of newly diagnosed type 2 diabetes: follow-up of a randomized trial. Diabetes Care 2014;37:1824-30.

32 Esposito K, Maiorino MI, Ciotola M, et al. Effects of a Mediterranean-style diet on the need for antihyperglycemic drug therapy in patients with newly diagnosed type 2 diabetes: a randomized trial. Ann Intern Med 2009;151:306-14.

33 García-Gavilán JF, Bulló M, Canudas S, et al. Extra virgin olive oil consumption reduces the risk of osteoporotic fractures in the PREDIMED trial. Clin Nutr 2018;37:329-35.

34 de Lorgeril M, Salen P, Martin J-L, et al. Mediterranean dietary pattern in a randomized trial. Arch Intern Med 1998;158:1181.

35 de Lorgeril M, Renaud S, Mamelle N, et al. Mediterranean alphalinolenic acid-rich diet in secondary prevention of coronary heart disease. Lancet 1994;343:1454-9.

36 De Lorgeril M, Salen P, Martin JL, et al. Effect of a Mediterranean type of diet on the rate of cardiovascular complications in patients with coronary artery disease. Insights into the cardioprotective effect of certain nutriments. J Am Coll Cardiol 1996;28:1103-8.

37 Díaz-López A, Babio N, Martínez-González MA, et al. Mediterranean diet, retinopathy, nephropathy, and microvascular diabetes complications: a post hoc analysis of a randomized trial. Diabetes Care 2015;38:2134-41.

38 Díaz-López A, Babio N, Martínez-González MA, et al. Erratum. Mediterranean Diet, Retinopathy, Nephropathy, and Microvascular Diabetes Complications: A Post Hoc Analysis of a Randomized Trial. Diabetes Care 2015;38:2134-2141. Diabetes Care 2018;41:2260-1.

39 Salas-Salvadó J, Bulló M, Babio N, et al. Erratum. Reduction in the Incidence of Type 2 Diabetes With the Mediterranean Diet: Results of the PREDIMED-Reus nutrition intervention randomized trial. Diabetes Care 2011;34:14-19. Diabetes Care 2018;41:2259-60.

40 García-Layana A, Ciufo G, Toledo E, et al. The effect of a Mediterranean diet on the incidence of cataract surgery. Nutrients 2017;9:453.

41 Gené Huguet L, Navarro González M, Kostov B, et al. Pre frail 80: multifactorial intervention to prevent progression of Pre-Frailty to frailty in the elderly. J Nutr Health Aging 2018;22:1266-74.

42 Marcos-Forniol E, Meco JF, Corbella E, et al. Secondary prevention programme of ischaemic heart disease in the elderly: a randomised clinical trial. Eur J Prev Cardiol 2018;25:278-86.

43 Martínez-González Miguel Á., Toledo E, Arós F, et al. Extravirgin olive oil consumption reduces risk of atrial fibrillation. Circulation 2014;130:18-26.

44 Papadaki A, Martínez-González MÁ, Alonso-Gómez A, et al. Mediterranean diet and risk of heart failure: results from the PREDIMED randomized controlled trial. Eur J Heart Fail 2017:19:1179-85

45 Properzi C, O'Sullivan TA, Sherriff JL, et al. Ad libitum Mediterranean and low-fat diets both significantly reduce hepatic steatosis: a randomized controlled trial. Hepatology 2018;68:1741-54

46 Salas-Salvadó J, Bulló M, Babio N, et al. Reduction in the incidence of type 2 diabetes with the Mediterranean diet: results of the PREDIMED-Reus nutrition intervention randomized trial. Diabetes Care 2011:34:14-19.

47 Salas-Salvadó J, Bulló M, Estruch R, et al. Prevention of diabetes with Mediterranean diets. Ann Intern Med 2014;160:1-10-10.

48 Salas-Salvadó J, Bulló M, Estruch R, et al. Prevention of diabetes with Mediterranean diets. Ann Intern Med 2018;169:271.

49 Singh RB, Dubnov G, Niaz MA, et al. Effect of an IndoMediterranean diet on progression of coronary artery disease in high risk patients (Indo-Mediterranean diet heart study): a randomised single-blind trial. Lancet 2002;360:1455-61.

50 Toledo E, Salas-Salvadó J, Donat-Vargas C, et al. Mediterranean diet and invasive breast cancer risk among women at high cardiovascular risk in the PREDIMED trial: a randomized clinica trial. JAMA Intern Med 2015;175:1752.

51 Tuttle KR, Shuler LA, Packard DP, et al. Comparison of low-fat versus Mediterranean-style dietary intervention after first myocardial infarction (from the heart Institute of Spokane diet intervention and evaluation trial). Am J Cardiol 2008;101:1523-30.

52 Pintó X, Fanlo-Maresma M, Corbella E, et al. A Mediterranean diet rich in extra-virgin olive oil is associated with a reduced prevalence of nonalcoholic fatty liver disease in older individuals at high cardiovascular risk. J Nutr 2019;149:1920-9.

53 García-Gavilán JF, Bulló M, Camacho-Barcia L, et al. Higher dietary glycemic index and glycemic load values increase the risk of osteoporotic fracture in the PREvención con Dleta MEDiterránea (PREDIMED)-Reus trial. Am J Clin Nutr 2018;107:1035-42.

54 Ruiz-Canela M, Estruch R, Corella D, et al. Association of Mediterranean diet with peripheral artery disease: the PREDIMED randomized trial. JAMA 2014;311:415-7.

55 Salas-Salvadó J, Fernández-Ballart J, Ros E, et al. Effect of a Mediterranean diet supplemented with nuts on metabolic syndrome status. Arch Intern Med 2008;168:2449.

56 Sánchez-Villegas A, Martínez-González MA, Estruch R, et al. Mediterranean dietary pattern and depression: the PREDIMED randomized trial. BMC Med 2013;11:208.

57 Greenberg I, Stampfer MJ, Schwarzfuchs D, et al. Adherence and success in long-term weight loss diets: the dietary intervention randomized controlled trial (direct). J Am Coll Nutr 2009;28:159-68.

58 Al Wattar BH, Dodds J, Placzek A, et al. Mediterranean-style diet in pregnant women with metabolic risk factors (Esteem): a pragmatic multicentre randomised trial. PLOS Med 2019;16:e1002857.

59 Assaf-Balut C, García de la Torre N, Durán A, et al. A Mediterranean diet with additional extra virgin olive oil and pistachios reduces the incidence of gestational diabetes mellitus (GDM): a randomized controlled trial: the St. Carlos GDM prevention study. PLoS One 2017;12:e0185873.

60 Assaf-Balut C, García de la Torre N, Duran A, et al. A Mediterranean diet with an enhanced consumption of extra virgin olive oil and Pistachios improves pregnancy outcomes in women without gestational diabetes mellitus: a Sub-Analysis of the St. Carlos gestational diabetes mellitus prevention study. Ann Nutr Metab 2019;74:69-79.

61 Babio N, Toledo E, Estruch R, et al. Mediterranean diets and metabolic syndrome status in the PREDIMED randomized trial. CMAJ 2014;186:E649-57.

62 de Lorgeril M, Salen P, Martin J-L, et al. Mediterranean diet, traditional risk factors, and the rate of cardiovascular complications after myocardial infarction. Circulation 1999;99:779-85.

63 Papadaki A, Martínez-González MÁ, Alonso-Gómez A, et al. Mediterranean diet and risk of heart failure: results from the PREDIMED randomized controlled trial. Eur J Heart Fail 2017;19:1179-1185. doi: 10.1002/ejhf.750. Eur J Heart Fail 2019;21:389-91.

64 Schulz KF, Altman DG, Moher D, et al. Consort 2010 statement: updated guidelines for reporting parallel group randomised trials. BMJ 2010;340:c332.

65 Fried LP, Tangen CM, Walston J, et al. Frailty in older adults: evidence for a phenotype. J Gerontol A Biol Sci Med Sci 2001;56:M146-57.

66 Schröder H, Fitó M, Estruch R, et al. A short screener is valid for assessing Mediterranean diet adherence among older Spanish men and women. $J$ Nutr 2011;141:1140-5. 
67 Al Wattar BH, Dodds J, Placzek A, et al. Effect of simple, targeted diet in pregnant women with metabolic risk factors on maternal and fetal outcomes (Esteem): study protocol for a pragmatic multicentre randomised trial. BMJ Open 2016;6:e013495.

68 American Diabetes Association. 5. Lifestyle Management: Standards of Medical Care in Diabetes-2019. Diabetes Care 2019;42:S46-60.

69 Franz MJ, MacLeod J, Evert A, et al. Academy of nutrition and dietetics nutrition practice guideline for type 1 and type 2 diabetes in adults: systematic review of evidence for medical nutrition therapy effectiveness and recommendations for integration into the nutrition care process. J Acad Nutr Diet 2017;117:1659-79.

70 Mudaliar U, Zabetian A, Goodman M, et al. Cardiometabolic risk factor changes observed in diabetes prevention programs in US settings: a systematic review and meta-analysis. PLoS Med 2016;13:e1002095.

71 American Diabetes Association. 8. Obesity Management for the Treatment of Type 2 Diabetes: Standards of Medical Care in Diabetes-2020. Diabetes Care 2020;43:S89-97.

72 Galaviz KI, Weber MB, Straus A, et al. Global diabetes prevention interventions: a systematic review and network meta-analysis of the real-world impact on incidence, weight, and glucose. Diabetes Care 2018;41:1526-34.

73 Parmar MKB, Carpenter J, Sydes MR. More multiarm randomised trials of superiority are needed. Lancet 2014;384:283-4.

74 Correia LLC. Primary prevention of cardiovascular disease with a Mediterranean diet supplemented with extra-virgin olive oil or nuts. N Engl J Med 2018;379:1387-9.

75 Ros E. The PREDIMED study. Endocrinol Diabetes y Nutr 2017;64:63-6.

76 Pędziwiatr M, Mizera M, Wysocki M, et al. The fragility of statistically significant results from clinical nutrition randomized controlled trials. Clin Nutr 2020;39:1284-91.

77 Zeilstra D, Younes JA, Brummer RJ, et al. Perspective: fundamental limitations of the randomized controlled trial method in nutritional research: the example of probiotics. Adv Nutr 2018;9:561-71.

78 Heaney RP, Nutrients HRP. Nutrients, endpoints, and the problem of proof. J Nutr 2008;138:1591-5.

79 Hébert JR, Frongillo EA, Adams SA, et al. Perspective: randomized controlled trials are not a panacea for diet-related research. Adv Nutr 2016;7:423-32.

80 Satija A, Yu E, Willett WC, et al. Understanding nutritional epidemiology and its role in policy. Adv Nutr 2015;6:5-18.

81 Bertaggia L, Baiardo Redaelli M, Lembo R, et al. The fragility index in peri-operative randomised trials that reported significant mortality effects in adults. Anaesthesia 2019;74:1057-60.

82 Goerke K, Parke M, Horn J, et al. Are results from randomized trials in anesthesiology robust or fragile? an analysis using the fragility index. Int J Evid Based Healthc 2020;18:116-24.

83 Chin B, Copeland A, Gallo L, et al. The fragility of statistically significant randomized controlled trials in plastic surgery. Plast Reconstr Surg 2019;144:1238-45.

84 Vargas M, Buonano P, Marra A, et al. Fragility index in multicenter randomized controlled trials in critical care medicine that have shown reduced mortality. Crit Care Med 2020;48:e250-1.

85 Khormaee S, Choe J, Ruzbarsky JJ, et al. The fragility of statistically significant results in pediatric orthopaedic randomized controlled trials as quantified by the fragility index: a systematic review. $J$ Pediatr Orthop 2018;38:e418-23.
86 Robinson T, Al-Shahwani N, Easterbrook B, et al. The fragility of statistically significant findings from randomized controlled trials in pediatric appendicitis: a systematic review. J Pediatr Surg 2020;55:800-4.

87 McCambridge J, Witton J, Elbourne DR. Systematic review of the Hawthorne effect: new concepts are needed to study research participation effects. J Clin Epidemiol 2014;67:267-77.

88 Rubin R. Backlash over meat dietary recommendations raises questions about corporate ties to nutrition scientists. JAMA 2020;323:401.

89 Tsirou E, Grammatikopoulou MG, Theodoridis X, et al. Guidelines for Medical Nutrition Therapy in Gestational Diabetes Mellitus: Systematic Review and Critical Appraisal. J Acad Nutr Diet 2019;119:1320-39.

90 Johnston BC, Alonso-Coello P, Bala MM, et al. Methods for trustworthy nutritional recommendations NutriRECS (Nutritional Recommendations and accessible Evidence summaries Composed of Systematic reviews): a protocol. BMC Med Res Methodol 2018;18:162.

91 Johnston A, Kelly SE, Hsieh S-C, et al. Systematic reviews of clinical practice guidelines: a methodological guide. J Clin Epidemiol 2019;108:64-76.

92 Majeed MB, Agrawal R, Attar B. Fragility Index of Randomized Controlled Trials in American College of Gastroenterology Guidelines on the Management of Crohn's Disease. Gastroenterology 2020;32:193-8.

93 Acuna SA, Sue-Chue-Lam C, Dossa F. The fragility Index-P values Reimagined, flaws and all. JAMA Surg 2019;154:674.

94 Kohlmeier M. Overblown claims. BMJ Nutr Prev Health 2018;1:5-6.

95 Trichopoulou A, Martínez-González MA, Tong TY, et al. Definitions and potential health benefits of the Mediterranean diet: views from experts around the world. BMC Med 2014;12:112.

96 Martínez-González MA, Sánchez-Villegas A. The emerging role of Mediterranean diets in cardiovascular epidemiology: monounsaturated fats, olive oil, red wine or the whole pattern? Eur $J$ Epidemiol 2004;19:9-13.

97 Davis C, Bryan J, Hodgson J, et al. Definition of the Mediterranean diet; a literature review. Nutrients 2015;7:9139-53.

98 Juszczak E, Altman DG, Hopewell S, et al. Reporting of Multi-Arm parallel-group randomized trials. JAMA 2019;321:1610.

99 Schaefer EJ, Augustin JL, Schaefer MM, et al. Lack of efficacy of a food-frequency questionnaire in assessing dietary macronutrient intakes in subjects consuming diets of known composition. Am J Clin Nutr 2000;71:746-51.

100 Mahabir S, Baer DJ, Giffen C, et al. Calorie intake misreporting by diet record and food frequency questionnaire compared to doubly labeled water among postmenopausal women. Eur J Clin Nutr 2006;60:561-5.

101 Mozaffarian D, Forouhi NG. Dietary guidelines and health-is nutrition science up to the task? BMJ 2018;360:k822.

102 Andrade $\mathrm{C}$. The use and limitations of the fragility index in the interpretation of clinical trial findings. J Clin Psychiatry 2020;81:20f13334.

103 Dettori JR, Norvell DC. How fragile are the results of a trial? the fragility index. Global Spine J 2020;10:940-2.

104 Carter RE, McKie PM, Storlie CB. The fragility index: a P-value in sheep's clothing? Eur Heart J 2017;38:346-8.

105 Charilaou P. Walking on thin ice: considering the fragility index in randomized control trials. Eur J Gastroenterol Hepatol 2020;32:139. 